\title{
CULTIVO E POTENCIAL USO DE PLANTAS FRUTíFERAS EM QUINTAIS URBANOS, POCONÉ, PANTANAL MATOGROSSENSE
}

\author{
CULTIVATION AND POTENTIAL USE OF FRUIT PLANTS IN BACKYARD URBAN \\ POCONÉ, PANTANAL MATOGROSSENSE
}

\section{CULTIVO Y USO POTENCIAL DE PLANTAS DE FRUTAS EN PATIOS URBANOS POCONÉ, MATOGROSENSIÓN PANTANAL}

\section{Resumo}

Adriana de Souza e Silva

Ezequiel da Costa Ferreira ${ }^{2}$

Rodrigo Ferreira de Morais ${ }^{3}$

Fernando Ferreira de Morais ${ }^{4}$

Este trabalho teve por objetivo realizar o levantamento das plantas frutíferas em quintas em Poconé, Pantanal de Mato Grosso e, por meio deste levantamento responder as seguintes questões: 1 - Quais as estruturas morfológicas das planas frutíferas cultivadas nos quintais são utilizadas pelos informantes; 2 - Existe um relação entre riqueza de espécies e o tamanho $\left(\mathrm{m}^{2}\right)$ do quintal? 3 - Idade do quintal e tempo de ocupação do quintal podem influenciar no número de espécies? Utilizamos técnicas de entrevista estruturada e semiestruturada, para obtenção dos dados socioeconômicos e levantamento das espécies frutíferas. Para verificar a relação do tamanho $\left(\mathrm{m}^{2}\right)$, idade dos quintais e tempo de ocupação do quintal com a riqueza de plantas frutíferas, realizamos uma análise regressão simples. Levantamos 55 espécies e 94,5\% indicadas para uso alimentar, seja in natura ou feito doces, sucos e geleias. Na análise de regressão linear simples observamos que a área $\left(\mathrm{m}^{2}\right)$, idade do quintal e tempo de moradia do entrevistado no quintal não influenciaram na riqueza de espécies frutíferas. Os quintais, independentemente do tamanho, idade ou do tempo de ocupação exercem um papel importante por contribuir no sustento familiar, uma vez que a maioria das espécies são usadas na alimentação dos familiares.

Palavras chave: agroecossistemas, botânica econômica, riqueza.

\footnotetext{
1 Universidade do Estado de Mato Grosso. Programa Parceladas. Campus Cáceres - MT.

2 Prodema-Doutorado - Dep. de Sistemática e Ecologia-Universidade Federal da Paraíba, Campus I, ORCID id: oooo-0003-4758-7171

3 Universidade Estadual do Piauí, Campus Dep. Jesualdo Cavalcanti, Corrente, PI.

Orcid id: oooo-ooo1-8687-3467

4 Departamento de Sistemática e Ecologia, CCEN, Universidade Federal da Paraíba, Campus Universitário I, CEP 58051-90o, João Pessoa, PB, Brasil. Email - fernandobotanica@gmail.com. Orcid id: oooo-ooo1-7111-2833
} 


\begin{abstract}
This work had the objective of performing the survey of the fruit trees in backyards in the Pantanal of Mato Grosso and, using the survey to answer the following questions: 1 - Which morphological structures of the fruit trees cultivated in the backyards are used by the informants; 2 - Is there a relation between the richness of species and the size ( $\mathrm{sm}$ ) of the backyard? 3 - Age of the backyard and time of occupation of the backyard can influence in the number of species? The techniques of structured and semistructured interview were used, for obtaining social economic data and survey of the fruit species. To check the size relation (sm), age of the backyards and time of occupation of the backyard with the richness of the fruit trees, we performed a simple regression analysis. Whe found 55 species and $94,5 \%$ were indicated for eating, either in natura or like sweets, juices. In the analysis of simple linear regression we observed that the area $(\mathrm{m})$ of the, age of the backyard and time of residence of the interviewee in the backyard did not influence the richness of fruit species. The backyards, despite the size, age or of the time of occupation perform an important role to contribute in family sustenance, as the majority of the species are used in the alimentation of the members of the family.
\end{abstract}

Keywords: agroecosystems, economy botany, richness.

\title{
Resumen
}

Este trabajo tuvo como objetivo encuestar las plantas frutales en las granjas de Poconé, Pantanal de Mato Grosso y, a través de esta encuesta, responder las siguientes preguntas: 1 - ¿Cuáles son las estructuras morfológicas de los árboles frutales cultivados en los patios? 2 - ¿Existe una relación entre la riqueza de especies y el tamaño (m2) del patio? 3 - ¿La edad del patio y el tiempo de ocupación del patio pueden influir en el número de especies? Utilizamos técnicas de entrevista estructuradas y semiestructuradas para obtener datos socioeconómicos y encuestas de especies frutales. Para verificar la relación del tamaño (m2), la edad de los patios y el tiempo de ocupación del patio con la riqueza de las plantas frutales, realizamos un análisis de regresión simple. Se encuestó a 55 especies y el 94,5\% indicado para uso alimentario, ya sea in natura o hecho de dulces, jugos y jaleas. En el análisis de regresión lineal simple observamos que el área (m2), la edad del patio y el tiempo de residencia del encuestado en el patio no influyeron en la riqueza de las especies frutales. Los patios traseros, independientemente del tamaño, la edad o el tiempo de ocupación, juegan un papel importante para contribuir al sustento familiar, ya que la mayoría de las especie se utilizan para alimentar a los miembros de la familia

Palabras clave: agroecosistemas, botánica económica, riqueza. 


\section{INTRODUÇÃO}

As plantas possuem sempre uma grande importância em muitas culturas e são utilizadas para suprir necessidades básicas, como alimentação, vestuário, moradia e para fins medicinais (Vitalini et al., 2009). A existência atual de muitas sociedades deve-se às conquistas dos seus ancestrais e, sem dúvida, entre elas está o manejo da diversidade vegetal (Thomas e Van Damme, 2010).

Para Guarim Neto e Maciel (2008) os recursos vegetais têm uma importância essencial, seja como alimento, matéria prima ou até como significado cultural. Esses autores relatam ainda que os recursos naturais e as plantas cultivadas dos quais a humanidade dependem, devem ser compreendidos por meio de estudos acadêmicos, onde as técnicas de manejo do ambiente realizadas por populações tradicionais ou não tradicionais poderão ser explicadas. Em Mato Grosso, por exemplo, há esforços no sentido de caracterizar e demonstrar a importância dos quintais para as populações pantaneiras: (Santos e Carnielo 2017; Carnielo et al. 2010; Guarim Neto e Nunes do Amaral 2010; Guarim Neto, 2008).

O manejo, a coleta e o consumo da vegetação são documentados em vários contextos culturais e ocorrem desde em áreas de agricultura intensiva até áreas mais intocadas, como as florestas, ilustrando a vasta importância e utilização das plantas em muitas sociedades humanas (Cruz-Garcia e Price, 2011).

Diante do exposto, os estudos etnoecológicos têm experimentado uma expressiva evolução no contexto das ciências naturais nas últimas décadas, constituindo um campo relativamente novo da ciência: a 'etnociência', que instala seu objeto de estudo e método na fronteira entre as ciências naturais e sociais (Costa 2008). Uma grande contribuição da etnociência pode ser constatada através do crescente número de trabalhos sobre o conhecimento das populações tradicionais e suas relações com o meio ambiente, indicando vários elementos úteis para o desenvolvimento de uma região (Alves et al., 2010). Assim, a etnoecologia surge como uma ferramenta que possibilitou entender melhor as complexas relações ser humano com a natureza, em grupos humanos que convivem mais diretamente com o ambiente natural (Grando 2007), baseado nas experiências de gerações passadas (Camargo et al. 2014).

Pesquisas sobre o manejo de ecossistemas estão relacionadas com o conhecimento entre as populações e o seu ambiente (Cunha et al. 2007). Conhecer o costume pelo qual diferentes sociedades humanas se relacionam com o ambiente circundante, tem papel fundamental à medida que na busca do conhecimento popular, esses saberes vão sendo armazenados e sistematizados (Santos 2004). A relação do homem com o componente vegetal, pode se dar no ambiente natural, ou em um ambiente modificado de onde moram. Neste contexto, os locais onde essa relação acontece, recebem várias definições, por diversos autores.

O termo 'conhecimento ecológico tradicional' (CET) pode ser utilizado para se referir ao corpo cumulativo de conhecimento, prática e crença, que evolui por meio 
de processos adaptativos e passados através das gerações por Transmissão cultural, acerca das relações entre os seres vivos e com seu ambiente (Berkes et al., 2000). Neste contexto, a etnobotânica possibilita os estudos das interações e relações entre plantas e pessoas no tempo e no espaço, o que inclui o uso, conhecimento, crenças, sistemas de gestão, classificação e linguagem, tanto nas culturas modernas quanto nas tradicionais (Kauai Declaration, 2007).

A região pantaneira apresenta uma realidade socioeconômica e ambiental ainda pouco conhecida, no que se refere, principalmente, à forma como as populações locais se relacionam com os recursos naturais ali existentes, em suas múltiplas dimensões (Reis e Guarim Neto 2000), dado o pequeno número de estudos etnobotânicos observados para essa região em comparação a outras regiões do Brasil (Ritter et al 2015).

A relação do homem com o reino vegetal é complexa, podendo ser considerada através de várias perspectivas, sendo que, uma delas, a mais visível e importante, refere-se à dependência direta ou indireta dos seres humanos em relação às plantas, que são necessárias para a sua sobrevivência (Amorozo 2010). Neste sentido, um dos conceitos aplicados aos locais de interação homem planta, explica que esses espaços têm uma demarcação diferenciada e depende do contexto da comunidade que está estudando, assim, o quintal é o espaço situado na parte posterior das residências, que na maioria das vezes são utilizados para o plantio de espécies, com certa finalidade de uso, cuja sua função é caracterizada de acordo com que os moradores de um determinado local destinam para esses espaços (Guarim Neto, 2008).

Em relação ao termo quintal, pode ser considerado como sistemas agroflorestais que resultam em benefícios nutricionais, econômicos e ecológicos, onde são introduzidas também novas espécies, devido ao transporte de sementes ou mudas de quintais vizinhos e de outras localidades, permitindo desta forma a manutenção da diversidade desses recursos vegetais (Fracaro 2003). Os quintais podem ser tidos ainda como espaços de uso, manejo e conservação de diferentes espécies vegetais contribuindo para a renda familiar e segurança alimentar das famílias (Spiller et al. 2016; Panyadee et al. 2016).

Os quintais são considerados uma das formas mais antigas de manejo de terra, o qual indica a sua sustentabilidade (Amaral e Guarim Neto 20o8). Desta forma, esses espaços apresentam importância fundamental, pois, ao cultivar plantas alimentícias, contribui na alimentação das famílias de baixa renda (Valadão 2006; Whitney et al. 2018). Sendo assim, os quintais podem ser de grande importância para a região de estudo, por ser uma das maneiras de subsidiar os alimentos necessários no dia a dia, além de contribuir na economia familiar, evitando a compra no comércio local daquilo que é produzido no quintal.

Este trabalho teve por objetivo realizar o levantamento das plantas frutíferas em quintas do município de Poconé, no Pantanal de Mato Grosso e, por meio deste levantamento responder as seguintes questões: 1 - Quais as estruturas morfológicas das planas frutíferas cultivadas nos quintais são utilizadas pelos 
informantes? 2 - Existe relação entre riqueza de espécies e o tamanho $\left(\mathrm{m}^{2}\right)$ do quintal? 3 - A Idade do quintal e tempo de ocupação do quintal podem influenciar no número de espécies?

\section{MATERIAL E MÉTODOS}

\section{Área de estudo}

As amostragens foram realizadas no bairro João Godofredo com as

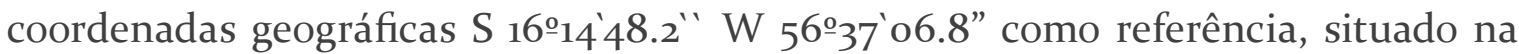
periferia do município de Poconé (figura o1). O município de Poconé está localizado no Pantanal Mato-Grossense, distante da capital Cuiabá a $95 \mathrm{~km}$ tendo uma extensão territorial de $17.126,38 \mathrm{~km}^{2}$, com o clima Tropical quente e sub-úmido, precipitação média anual de $1.500 \mathrm{~mm}$, com intensidade máxima em dezembro, janeiro e fevereiro, tendo temperatura média anual de $24^{\circ} \mathrm{C}$, máxima $42^{\circ} \mathrm{C}$ e mínima $4^{\circ} \mathrm{C}$. (Governo do Estado de Mato Grosso, 2010).

A vegetação dessa região constitui-se de formações arbustivas e herbáceas, sendo típica do pantanal de Cerrado e de campos inundáveis (Fonseca 2008).

A seleção do domicilio para realização da coleta de informações foi realizada por meio de sorteio. Considerando que o bairro possui 31 ruas, foi sorteada uma residência por rua, no entanto duas ruas não possuíam residências, assim, totalizamos 29 residências sorteadas para o contato com os moradores. O bairro estudado é considerado uns do mais populosos no município de Poconé com 13\% da população local, constituindo em um total de 4.789 pessoas. Conta com uma escola municipal, que atende a comunidade local e bairros circunvizinhos. 

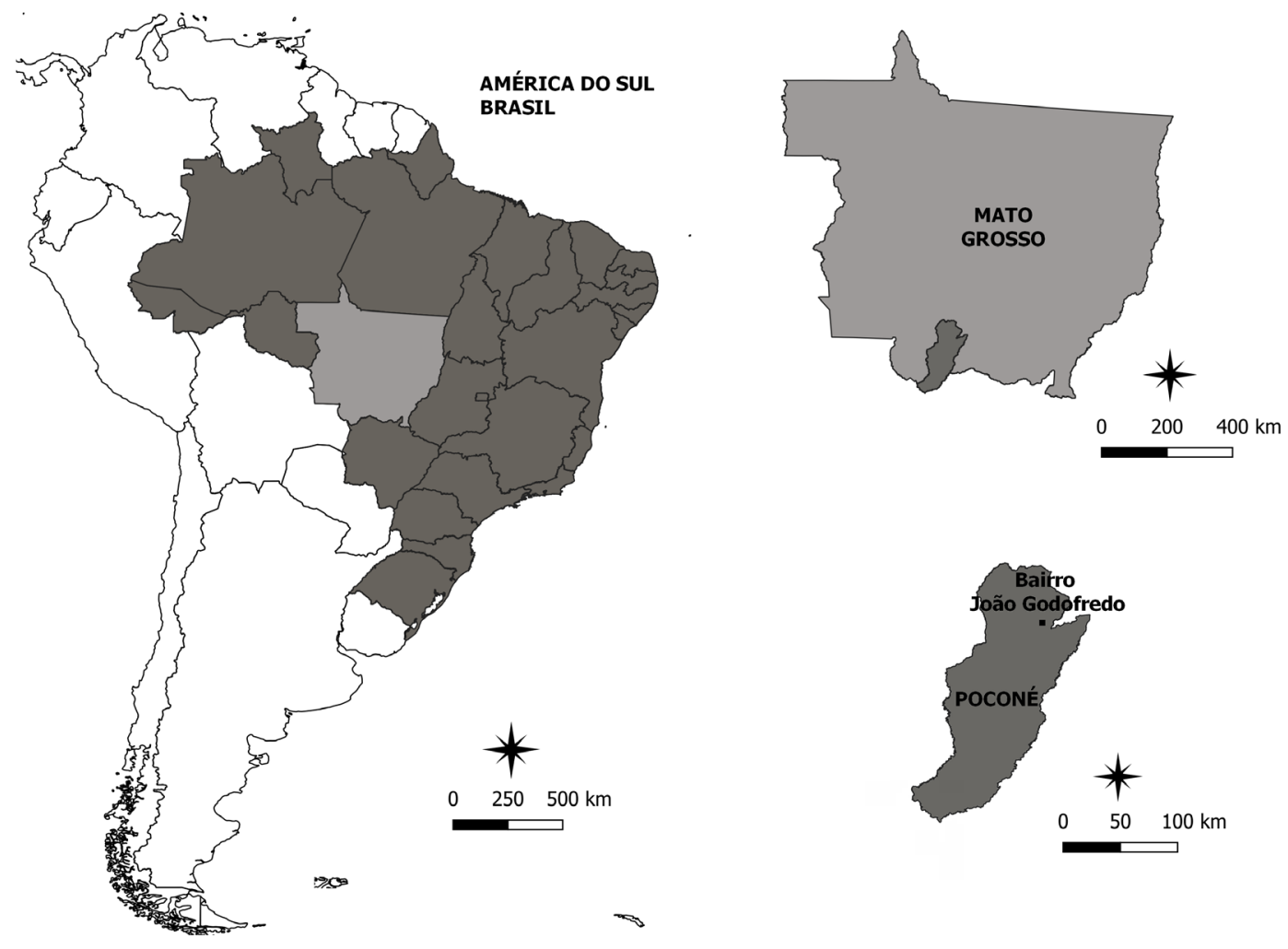

Figura o1. Bairro João Godofredo, Município de Poconé, Pantanal de Mato Grosso.

Esta pesquisa fundamentou-se qualitativa e quantitativamente, com intuito de investigar quais as plantas frutíferas que esta população conhece e cultiva em seus quintais. É importante ressaltar que a pesquisa qualitativa preocupa-se em esclarecer como a cultura em questão compreende o mundo, nesse estudo, as plantas frutíferas dos quintais. No caso da obtenção de dados quantitativos, podemos proceder análises e comparações com outros estudos no mesmo contexto (Albuquerque el al. 2017).

Nesta pesquisa foi utilizado o método de entrevista, que é considerado uma forma de interação social, mais especificamente, é uma forma de diálogo assimétrico, em que uma das partes busca coletar dados e a outra se apresenta como fonte de informação, sendo bastante adequada para a obtenção de informações acerca do que as pessoas sabem e creem (Gil 2009). A entrevista pode se distingui por uma simples conversação ou por uma lista de perguntas chamada de questionário, sendo uma técnica de investigação composta de perguntas destinadas a pessoas, com o objetivo de coleta de dados sobre conhecimentos, crenças, valores, entre outros (Gil 2009; Andrade 2010).

Para obtenção de informações referentes aos moradores e às plantas frutíferas existentes nos quintais, realizamos entrevistas semiestruturadas (Albuquerque et al. 2014), técnica importante que permite o desenvolvimento de uma estreita relação entre o pesquisador e o entrevistado (Richardson 2008). A entrevista semiestruturada 
contribui para melhor percepção ambiental, cultural e social dos fenômenos locais, por meio das informações relacionadas ao ambiente e ao cotidiano da comunidade (Pasa 2004).

Para a coleta e herborização do material botânico foi usado o descrito por Fidalgo e Bononi (1984). Foram coletadas amostras de material fértil de todos os indivíduos e, a identificação foi realizada com auxílio de literatura especializada, consulta a especialista e comparações com a coleção do Herbário da Universidade Federal do Piauí, Graziela Barroso. O sistema de Classificação utilizado foi o APG (2016), e as sinonímias foram verificadas em BFG (2020).

A mensuração em $\mathrm{m}^{2}$ dos quintais foi de acordo à resposta dos entrevistados e para explorar as relações entre o tamanho dos quintis, idade dos quintais e tempo em que os moradores residem no local com a riqueza de plantas frutíferas dos quintais foi utilizada a análise de regressão linear simples $(\mathrm{P}<0,05)$.

\section{RESULTADOS}

Para caracterização da população estudada foram entrevistados 29 moradores, dentre estes 28 foram do sexo feminino e um do sexo masculino.

Conforme apresentado na (figura 2), as classes etárias com maior número de informantes ocorreu entre 26-30 anos cinco, 61 a 65 quatro, 66-70 anos seis, somando 15 pessoas. As classes etárias entre 21-25 anos e 71-75 verificamos apenas um informante em cada uma.

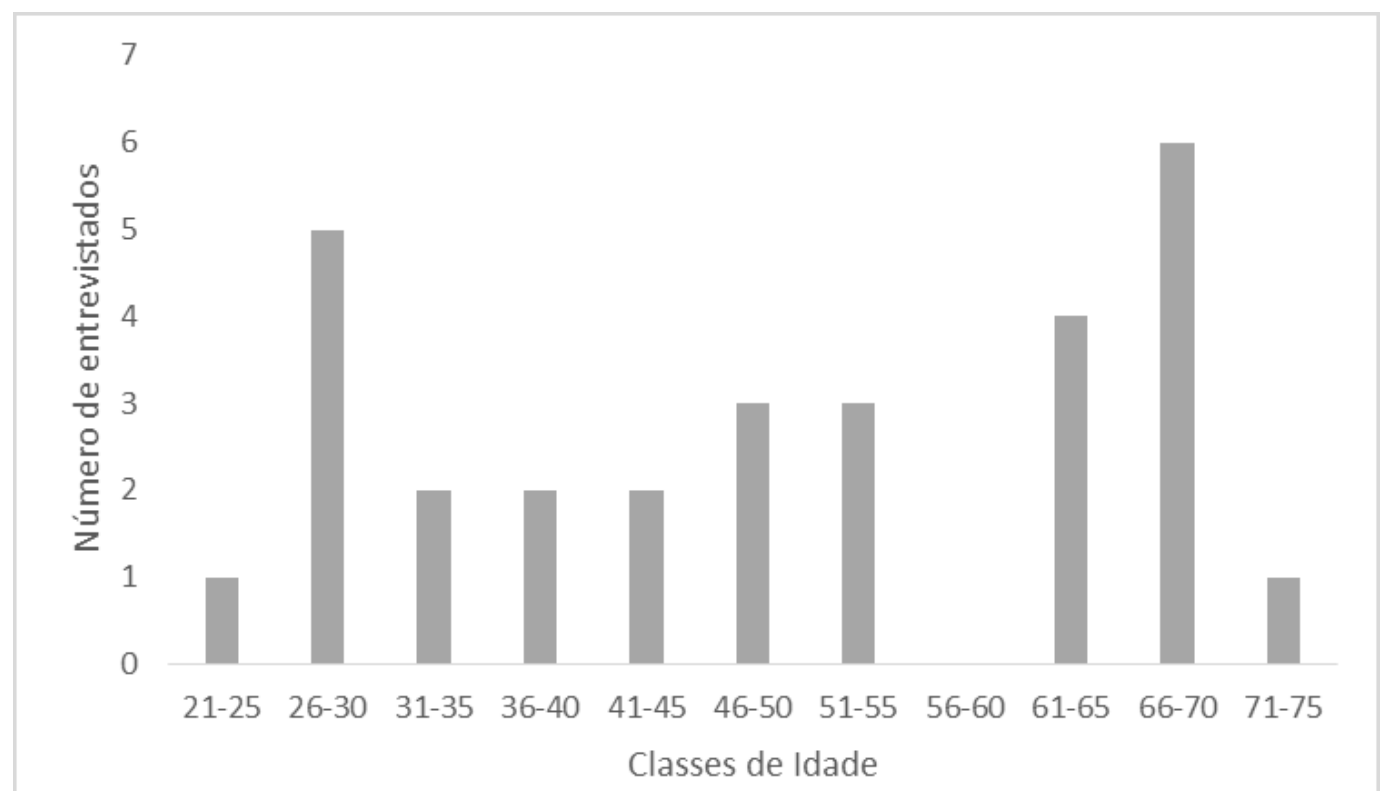

Figura 2. Classes de idades dos informantes no bairro João Godofredo, Poconé-MT. 
Com relação a ocupação dos informantes, verificamos que os mesmos se identificaram em sete categorias conforme podemos observar na (figura 3), sendo que em duas categorias, do lar e aposentados somaram $79 \%$ dos informantes, $48 \%$ do Lar e 31\% aposentados, neste grupo encontra-se o único senhor que participou da pesquisa, as demais categorias de ocupação somadas foram $21 \%$.

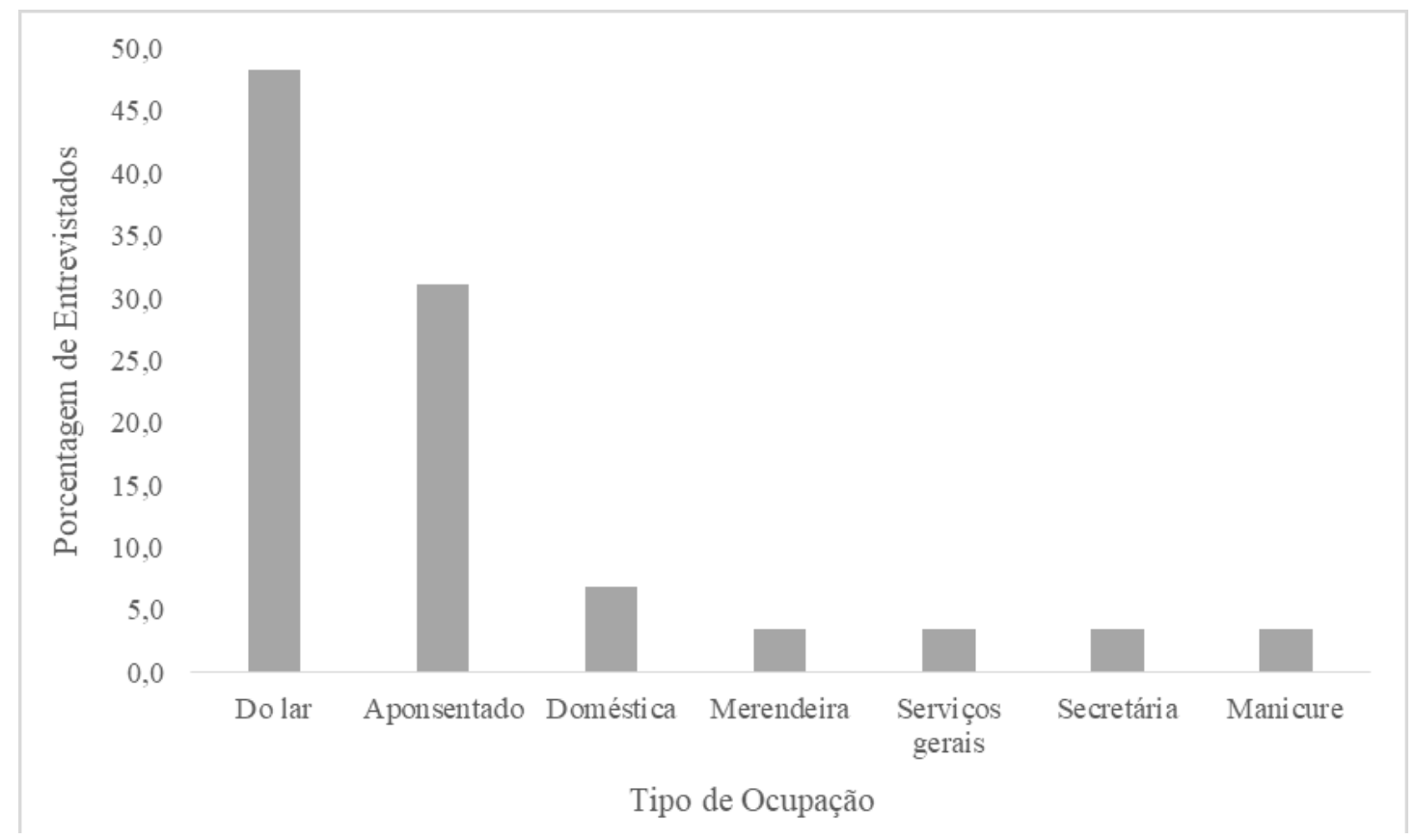

Figura 3. Tipo de ocupação dos informantes moradores no bairro João Godofredo, PoconéMT.

As plantas frutíferas encontradas nos quintais (tabela 1) estudados variaram de duas a 21 espécies por quintal, de forma que das 55 espécies frutíferas, algumas apresentaram mais de uma utilidade: $94,5 \%$ têm como finalidade atender as necessidades alimentares, seja in natura ou feito doces, sucos e geleias, seguido de 51\% medicinal, 3,6 \% para usos diversos (tempero, limpar peixe e isca para peixes,) e apenas 1,8 \% excelente para sombreamento no quintal. Como medicinal, apenas 5,5\% foram exclusivas. 
Tabela o1: Família botânica, espécies e categoria de uso encontrados nos quintais pesquisados do bairro João Godofredo, Poconé - MT.

\begin{tabular}{|c|c|c|c|c|}
\hline Família/ Espécie & Nome popular & Uso & Parte usada & Preparo \\
\hline \multicolumn{5}{|l|}{ Anacardiaceae } \\
\hline Anacardium occidentale L. & Caju amarelo, Caju de Metro e Caju Vermelho & A, M & Fruto, a casca do caule & $\begin{array}{l}\text { In-natura, suco, doce, rapadura, pé-de- } \\
\text { moleque, chá }\end{array}$ \\
\hline Mangifera indica $\mathbf{L}$. & Manga borbona (Bourbon), Manga cumbaru e Manga Rosa & A, M, S & $\begin{array}{l}\text { Fruto, a casca do caule, } \\
\text { Árvore }\end{array}$ & In-natura, doce, chá \\
\hline Spondias sp. & Cajá-manga & A & Fruto & In-natura \\
\hline Spondias purpurea L. & Jacote (ceriguela) & A, M & Fruto, a casca do caule & In-natura, suco, chá \\
\hline \multicolumn{5}{|l|}{ Annonaceae } \\
\hline Annona squamosa L. & Ata & A, M & Fruto, folhas & In-natura \\
\hline Annona sp. & Ataconde & A, M & Fruto, folhas & In-natura, chá \\
\hline Annona muricata L. & Graviola & $\mathrm{A}, \mathrm{M}$ & Fruto, folhas & In-natura, suco, chá \\
\hline \multicolumn{5}{|l|}{ Apocynaceae } \\
\hline $\begin{array}{l}\text { Hancornia speciosa var. pubescens } \\
\text { (Nees \& C. Mart.) Müll. Arg. }\end{array}$ & Mangava (Mangaba) & $\mathrm{A}, \mathrm{M}$ & Fruto, látex do caule & In-natura, doce \\
\hline \multicolumn{5}{|l|}{ Arecaceae } \\
\hline $\begin{array}{l}\text { Acrocomia aculeata (Jacq.) Lodd. } \\
\text { ex Mart. }\end{array}$ & Bocaiúva & $\mathrm{A}, \mathrm{M}$ & Fruto, estipe, raiz & $\begin{array}{l}\text { In-natura, farinha, sorvete, brigadeiro, } \\
\text { suco, Pé-de-moleque, chá }\end{array}$ \\
\hline Orbignya phalerata Mart. & Coco-aguaçu & A & Fruto & In-natura, doce \\
\hline Cocos nucifera L. & Coco amarelo e Coco da Baía & $\mathrm{A}, \mathrm{M}$ & Fruto & In-natura, cocada, bolo, doce \\
\hline \multicolumn{5}{|l|}{ Bignoniaceae } \\
\hline Crescentia cujete L. & Cabaça & $\mathrm{M}$ & Folhas & Chá \\
\hline \multicolumn{5}{|l|}{ Bromeliaceae } \\
\hline Ananas comosus (L.) Merr. & Abacaxi & $\mathrm{M}$ & Fruto & In-natura, suco, bolo \\
\hline
\end{tabular}




\begin{tabular}{|c|c|c|c|c|}
\hline Família/ Espécie & Nome popular & Uso & Parte usada & Preparo \\
\hline \multicolumn{5}{|l|}{ Caricaceae } \\
\hline Carica papaya L. & $\begin{array}{l}\text { Mamão castelo, Mamão Comum, Mamão Macho, Mamão } \\
\text { Papaia e Mamão Roxo }\end{array}$ & A, M & Fruto, flor & In-natura, doce, vitamina, salada, chá \\
\hline Caryocar brasiliense Cambess & Pequi & A & Fruto & In-natura \\
\hline \multicolumn{5}{|l|}{ Celastraceae } \\
\hline $\begin{array}{l}\text { Salacia elliptica (Mart. ex schult) } \\
\text { G. Don }\end{array}$ & Siputá & A & Fruto & In-natura \\
\hline \multicolumn{5}{|l|}{ Chrysobalanaceae } \\
\hline $\begin{array}{l}\text { Couepia uiti (Mart. \& Zucc) Benth. } \\
\text { ex Hook. F. }\end{array}$ & Pateiro & $\mathrm{A}, \mathrm{Ot}$ & Fruto & In-natura, isca para peixe \\
\hline \multicolumn{5}{|l|}{ Euphorbiaceae } \\
\hline Ricinus communis L. & Mamona & $\mathrm{M}$ & Fruto & Azeite \\
\hline \multicolumn{5}{|l|}{ Fabaceae } \\
\hline Dipteryx alata Vogel & Cumbaru & $\mathrm{A}, \mathrm{M}$ & Fruto & In-natura, chá ou coloca na água \\
\hline Inga sp. & Ingá & A & Fruto & In-natura \\
\hline Tamarindus indica L. & Tamarino & $\mathrm{A}, \mathrm{M}$ & Fruto, folhas & In-natura, suco, banho \\
\hline \multicolumn{5}{|l|}{ Lamiaceae } \\
\hline Vitex cymosa Bertero ex Spreng. & Tarumã & A & Fruto & In-natura \\
\hline \multicolumn{5}{|l|}{ Lauraceae } \\
\hline Persea americana Mill. & Abacate & $\mathrm{A}, \mathrm{M}$ & Fruto, folhas, sementes & In-natura, vitamina, chá \\
\hline \multicolumn{5}{|l|}{ Lythraceae } \\
\hline Punica granatum L. & Romã & $\mathrm{R}$ & Fruto & In-natura, chá, de molho na água \\
\hline \multicolumn{5}{|l|}{ Malpighiaceae } \\
\hline Malpighia glabra L. & Acerola & A, M & Fruto, folhas & In-natura, suco, geléia, chá \\
\hline
\end{tabular}




\begin{tabular}{|c|c|c|c|c|}
\hline Família/ Espécie & Nome popular & Uso & Parte usada & Preparo \\
\hline Bunchosia armeniaca (Cav.) DC. & Cereja & A & Fruto & Suco \\
\hline \multicolumn{5}{|l|}{ Malvaceae } \\
\hline Theobroma cacao L. & Cacau & A & Fruto & In-natura \\
\hline $\begin{array}{l}\text { Theobroma grandiflorum (Wiild. } \\
\text { Ex Spreng.) K. Schum. }\end{array}$ & Cupuaçu & A & Fruto & Suco \\
\hline \multicolumn{5}{|l|}{ Moraceae } \\
\hline Artocarpus sp. & Fruta- pão & A & Fruto & In-natura \\
\hline Artocarpus heterophyllus Lam. & Jaca & $\mathrm{A}, \mathrm{M}$ & Fruto, folhas & In-natura, suco, coloca a folha no álcool \\
\hline Morus nigra L. & Amora & $\mathrm{A}, \mathrm{M}$ & Fruto, folhas & In-natura, suco, chá \\
\hline Ficus carica L. & Figo & A & Fruto & Doce \\
\hline $\begin{array}{l}\text { Musaceae } \\
\text { Musa paradisiaca L. }\end{array}$ & $\begin{array}{l}\text { Banana d água, Banana-da-terra ou sartaveaco, Banana } \\
\text { Maçã, Banana Mariquita, Banana Nanica, Banana Ouro, } \\
\text { Banana Prata, Banana Rocha e Banana São Tomé }\end{array}$ & A, M & Fruto & $\begin{array}{l}\text { In-natura,doce, melado, vitamina, geléia, } \\
\text { rapadura }\end{array}$ \\
\hline \multicolumn{5}{|l|}{ Myrtaceae } \\
\hline Eugenia uniflora L. & Pitanga & A, M & Fruto, folhas & In-natura, suco, chá \\
\hline Psidium guajava L. & Goiaba branca, Goiaba vermellha e Goiaba pêra & A, M & Fruto, a casca do caule & $\begin{array}{l}\text { In-natura, suco, doce, chá ou coloca na } \\
\text { água }\end{array}$ \\
\hline Syzygium sp. & Jambo & A, M & Fruto & In-natura, chá \\
\hline Myrciaria sp. & Jabuticaba & A & Fruto & In-natura, suco, vinho \\
\hline \multicolumn{5}{|l|}{ Oxalidaceae } \\
\hline Averrhoa carambola L. & Carambola & A, M & Fruto, folhas & In-natura, suco, doce, chá \\
\hline \multicolumn{5}{|l|}{ Passifloraceae } \\
\hline Passiflora edulis Sims & Maracujá & A, M & Fruto & Suco, chá \\
\hline
\end{tabular}




\begin{tabular}{|c|c|c|c|c|}
\hline Família/ Espécie & Nome popular & Uso & Parte usada & Preparo \\
\hline \multicolumn{5}{|l|}{ Rubiaceae } \\
\hline Coffea arabica L. & Café & $\mathrm{A}, \mathrm{M}$ & Fruto, folhas & Café, coloca a folha no álcool \\
\hline Genipa americana L. & Jenipapo & $\mathrm{A}, \mathrm{M}$ & Fruto & In-natura \\
\hline \multicolumn{5}{|l|}{ Rutaceae } \\
\hline Citrus medica L. & Cidra & A & Fruto & Doce \\
\hline Citrus sp.1 & Laranja azeda & A & Fruto & Doce \\
\hline Citrus sp.2 & Laranja comum & $\mathrm{A}, \mathrm{M}$ & Fruto, folhas & In-natura, suco, abafado, chá \\
\hline Citrus sp.3 & Laranja misteriosa & $\mathrm{A}, \mathrm{M}$ & Fruto & Suco, doce \\
\hline Citrus sp.4 & Lima & A, M & Fruto, folhas & In-natura, chá \\
\hline Citrus sp.5 & Limão comum & A, M & Fruto & Suco, tempero, chá \\
\hline Citrus sp.6 & Limão galego & A & Fruto & Suco, tempero, doce, melado \\
\hline Citrus sp.7 & Limão rosa & $\mathrm{A}, \mathrm{Ot}$ & Fruto & Tempero, suco, limpar peixe \\
\hline Citrus sp.8 & Pocã & A & Fruto & In-natura \\
\hline Citrus sp.9 & Mexerica & A & Fruto & In-natura \\
\hline Citrus sp.10 & Tangerina & A & Fruto & In-natura \\
\hline \multicolumn{5}{|l|}{ Sapindaceae } \\
\hline $\begin{array}{l}\text { Talisia esculenta (A. St.- Hil.) } \\
\text { Radlk. }\end{array}$ & Pitomba & A & Fruto & In-natura \\
\hline \multicolumn{5}{|l|}{ Sapotaceae } \\
\hline Pouteria macrophylla (Lam.) Eyma & Fruta banana & A & Fruto & In-natura \\
\hline \multicolumn{5}{|l|}{ Vitaceae } \\
\hline Vitis vinifera $L$. & Uva & A & Fruto & In-natura \\
\hline
\end{tabular}

Legenda: $\mathrm{A}$ = Alimentar, $\mathrm{M}$ = Medicinal, $\mathrm{S}=$ =Sombreamento, $\mathrm{Ot}=$ Outros usos. 
Dentre as estruturas morfológicas das plantas mais indicadas pelos informantes, o destaque foi para o fruto, com $61,8 \%$, seguido do fruto e as folhas com $21,8 \%$, obtendo mais representatividade em relação as demais estruturas das plantas (Figura 04).

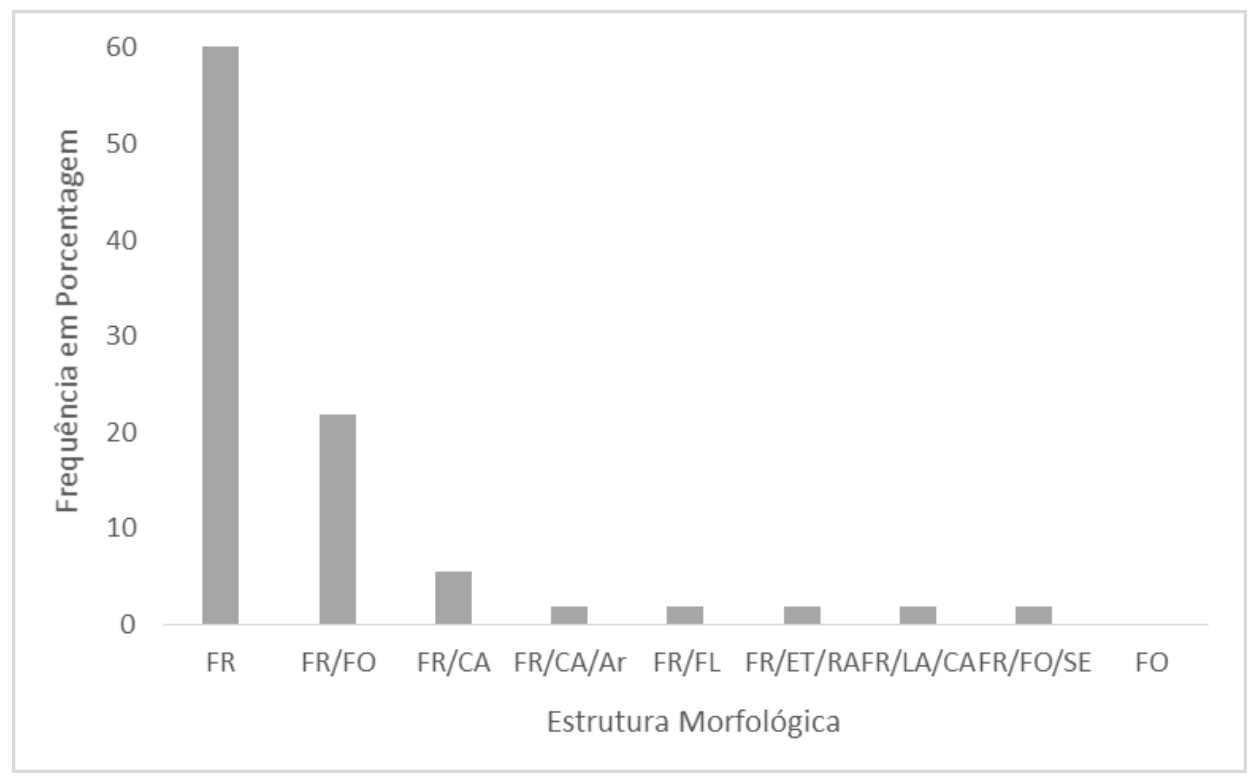

Figura 04. Valor percentual de estruturas morfológicas das plantas informado pelos entrevistados no bairro João Godofredo, Poconé-MT. (FR - Fruto; FO - Folhas; CA - Casca; AR - Árvore; FL - Flores; LA - Látex; SE - Semente; ET - Estipe).

Nossos resultados evidenciaram que a riqueza de espécies frutíferas não possui relação com o tamanho dos quintais $\left(\mathrm{R}^{2}=0,35\right)$ (figura 5 ).

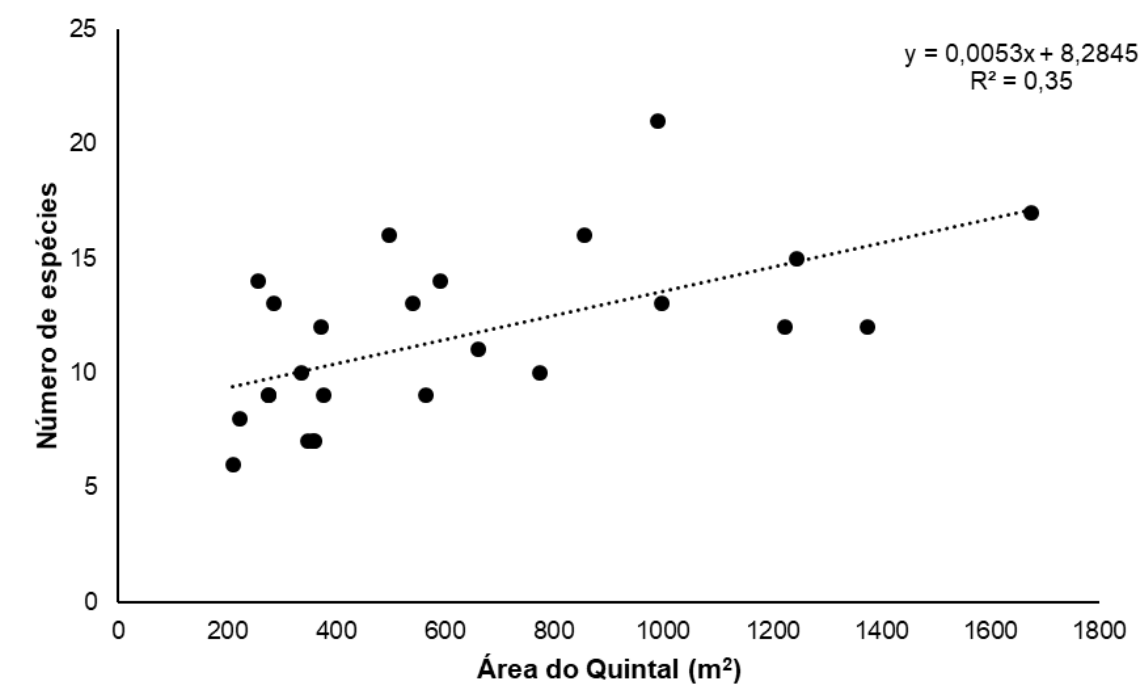

Figura 5. Relação da riqueza das espécies frutíferas com o tamanho dos quintais no bairro João Godofredo, Poconé-MT. 
Verificamos também que, e a riqueza das espécies frutíferas cultivadas nos quintais não possui relação com o tempo de moradia dos informantes $\left(\mathrm{R}^{2}=0,11\right)$ figura 6.

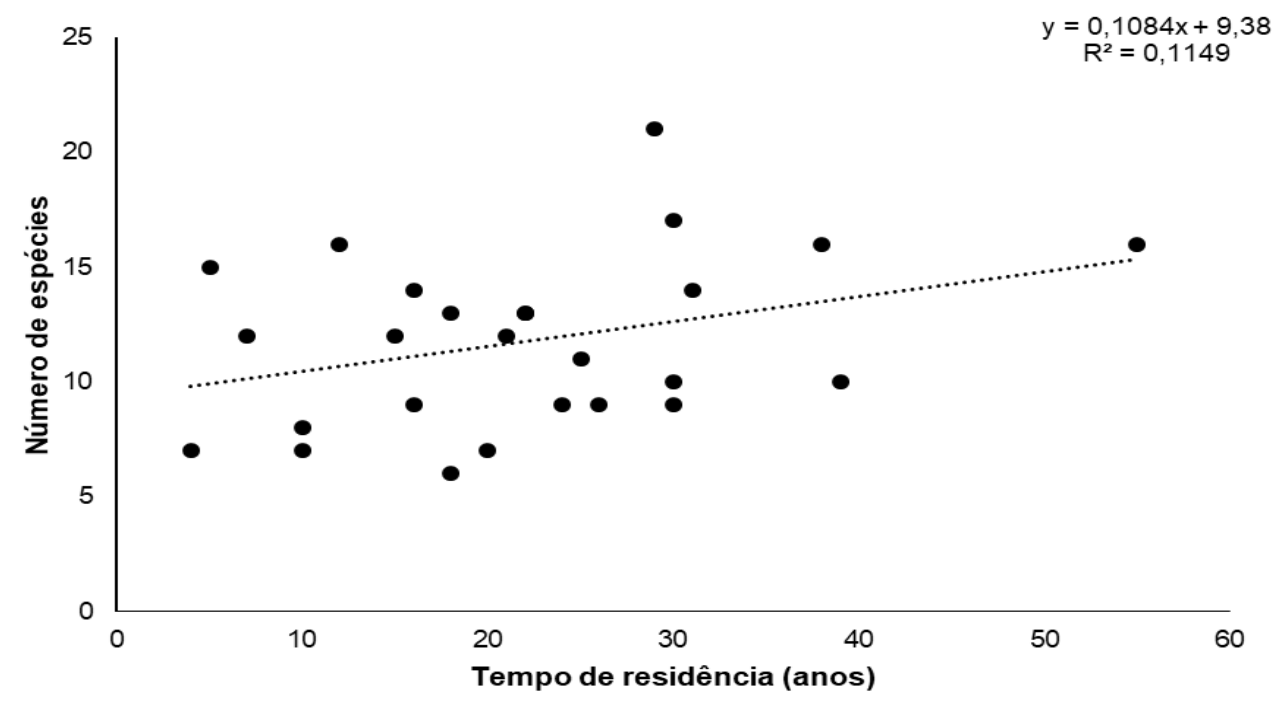

Figura 6. Relação da riqueza das espécies frutíferas encontradas nos quintais com o tempo de residência dos entrevistados do bairro João Godofredo, Poconé-MT.

Conforme observamos na figura 7 a idade do quintal, não apresentou relação com a riqueza de espécies frutíferas dos quintais $\left(\mathrm{R}^{2}=0,22\right.$ e $\left.\mathrm{p}>0,05\right)$.

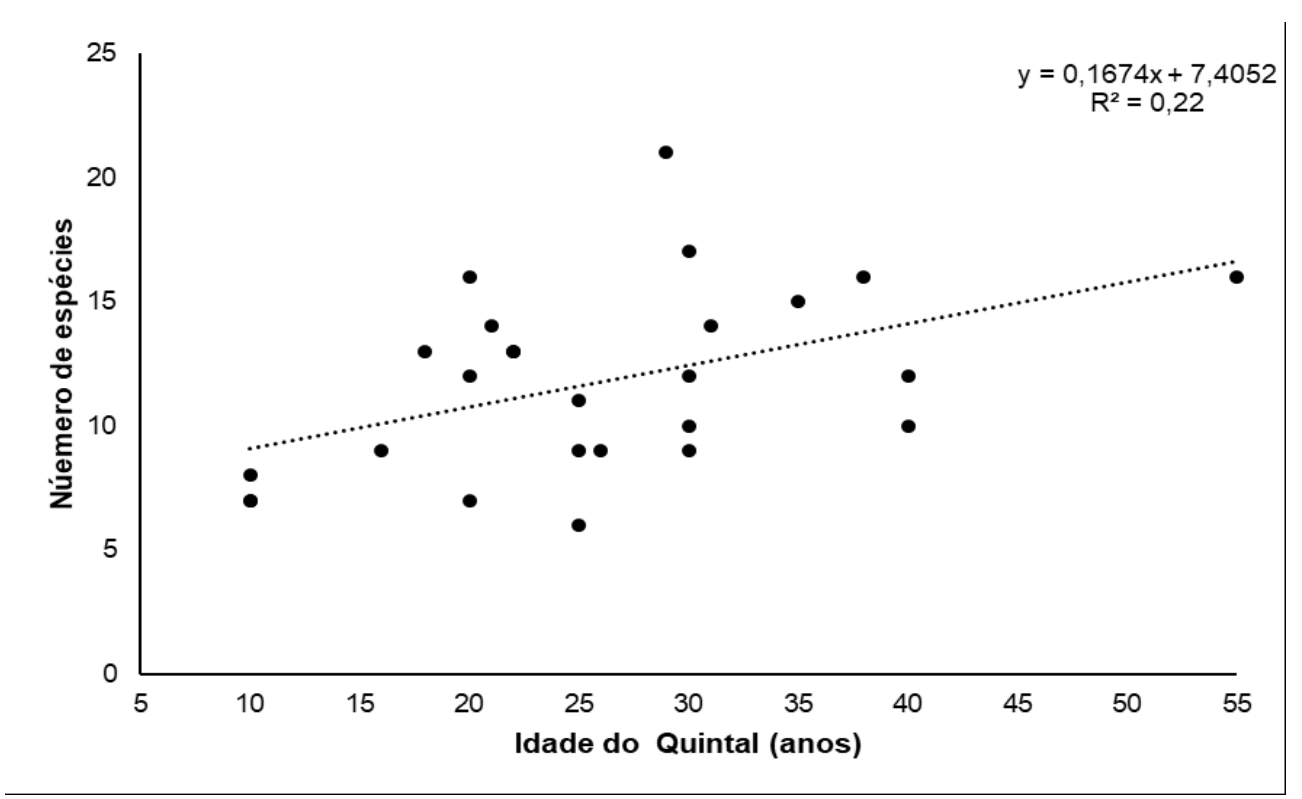

Figura 7. Relação da riqueza das espécies frutíferas com a idade dos quintais no bairro João Godofredo, Poconé-MT. 


\section{DISCUSSÃO}

Fica evidente que os informantes detém conhecimento sobre plantas frutíferas mesmo sendo moradores área urbana e o aprendizado se deu pela herança intergeracional, pois em vários relatos, os informantes disseram ter aprendido com pessoas mais velhas e da família.

Entre os participantes da pesquisa, as mulheres foram em maior número e tem papel importante no manejo de espécies vegetais nos quintais. Carniello et al. (2008) observa que as mulheres dedicam uma maior parte do seu tempo nos cuidados com o quintal, no qual produz e beneficia alimentos e remédios para sua família, gerando também excedentes para a comercialização. Pereira (2015) também observou que nos quintais Agroflorestais no Pantanal, as mulheres tem uma participação intensa no manejo de quase metade dos quintais. Os argumentos apresentados por Carniello et al. (2008) e Pereira (2015) ao maior número de mulheres nas pesquisas, também podem ser assumidos neste trabalho. Talvez o fato de apresentar mais mulheres no estudo, pode estar relacionado devido a ocupação dos informantes, pois mais da metade dos informantes são donas de casa e algumas são aposentadas, assim, preferencialmente tem mais tempo para ficar casa se dedicarem ao quintal. De acordo com Carniello et al. (2008), há ainda o costume de cultivar plantas nos quintais, preferencialmente frutíferas, pois a maioria das espécies são de uso na alimentação da família.

Nossos resultados demonstram com relação à idade dos informantes, a importância dos mais velhos para manutenção do conhecimento acerca das plantas frutíferas nos quintais. O maior número de pessoas com idade acima de 40 anos se deu pelo fato dos mais jovens justificarem que não contribuem no manejo do quintal e que pessoas com mais idade detinham maior conhecimento sobre o manejo e uso das plantas frutíferas. Vale ressaltar que de maneira geral os mais idosos conhecem uma diversidade maior de plantas úteis, devido ao saber acumulado ao longo de suas vidas (Hanazaki et al. 2003 e Tuler 2011). Para Salgado e Guido (2008) o maior conhecimento entre os mais velhos deve-se aos anos de relação e experiência em seu ambiente. O que de acordo com Marinho et al. (2011) contribui para esses desenvolverem uma capacidade muito própria de identificar as plantas, que inclui observações de aspectos morfológicos, ecológicos e culturais. Nas pesquisas realizadas por Carneiro et al. (2010), Silva (2010) e Pilla e Amorozo (2009), estes já apontaram que pessoas com mais idade são as mais representativas em estudos dessa natureza. Em campo observamos que os moradores mais idosos detentores do conhecimento, evidenciaram o desinteresse dos mais jovens.

Todos os informantes responderam ter plantas frutíferas em seus quintais, o que nos leva a afirmar que o cultivo de plantas frutíferas é comum nos quintais. Nossos resultados mostram também que a riqueza é comparável por exemplo ao apresentado por Rosa et al. (2007), a qual constataram que 51,3\% das espécies cultivadas em quintais no município de Bragança - PA, são frutíferas, e detêm a preferência dos agricultores familiares por contribuir na dieta da família. A riqueza 
das espécies levantadas e os depoimentos dos moradores evidenciam como as plantas frutíferas são importantes para a população estudada.

"Tem que plantar um pouco de cada, para não ter que comprar tudo." (SR a. I. moradora do bairro João Godofredo).

O fruto foi a estrutura morfológica mais indicada pelos informantes, haja vista, que é a estrutura mais comumente usada na alimentação. Guarin-Neto e Amaral (2010) observaram em quintais que a diversificação em relação às formas de uso das espécies destacando-se aquelas com fi nalidades medicinais (35\%), alimentares (33\%) e ornamentais (27\%). Também estudando quintais, Santos et al. (2019) indicaram que dentre as espécies (48) levantadas, 36 para fins de alimentação. Na mesma perspectiva, Moura e Andrade (2007) observaram em quintais no nordeste que dentre as espécies levantadas foram classificadas nas categorias alimentícias (52), ornamentais, (128), medicinais (53) e de valor comercial (2). Desta maneira, observamos que dentre as plantas comumente encontradas nos quintais as plantas alimentícias quanto não são as mais citadas, estão entre elas, pois desempenham papel importante na economia por não necessitar comprar e também na nutrição familiar pela diversidade de espécies encontradas nos quintais.

Observamos que o tamanho $\left(\mathrm{m}^{2}\right)$, tempo de moradia e idade dos quintais, não influenciaram na riqueza das espécies frutíferas. Entretanto, independentemente do tamanho, da idade quintal ou tempo em que o quintal esteja ocupado, os mesmos são considerados pelos moradores locais importantes, seja para obtenção de alimento, remédio, tempero ou mesmo conforto térmico. Observamos então que a potencialidade das plantas frutíferas de apresentar além de fonte alimentar, também recursos medicinais é de suma importância, pois dessa forma colabora com a renda da família por encontrar em sua própria residência plantas que servem para o preparo de remédios caseiros. Desta maneira, verificamos o quanto os quintais são importantes para os informantes. Spiller et al. (2016) aponta que as espécies usadas na alimentação são também as mais frequentes nos estudos sobre quintais no Brasil e Pasa (2007) constatou que $48 \%$ das espécies encontradas são de categoria alimentar, demonstrando o quintal como um importante fonte de alimento, tendo importância na complementação alimentar das famílias do local estudado. Os recursos vegetais encontrados nos quintais poderiam ser mais aproveitados pelos moradores do local, por meio do cultivo não só para o sustento familiar, mas também para a comercialização, visto que algumas áreas de quintais têm estrutura suficiente para o cultivo.

\section{CONCLUSÃO}

Nossa pesquisa revela o quão os quintais são importantes localmente e demonstra como a conservação desses espaços e do conhecimento associado, 
contribuem para preservação de conhecimentos tradicionais, que são imprescindíveis para a manutenção da identidade cultural nas comunidades. Também evidencia uma grande variedade de espécies de plantas frutíferas conhecidas e utilizadas para diversas finalidades, que de certa forma é conservada localmente, o que contribui para a manutenção do banco de germoplasma de espécies cultivadas.

Desta maneira, estudos realizados que visem conhecer o cultivo de plantas em quintais no Pantanal podem favorecer a tomada de decisões quanto às políticas de apoio à agricultura familiar e subsidiar recursos necessários para uma agricultura sustentável, favorecendo assim, a produção de plantas frutíferas para consumo o familiar e a comercialização.

\section{REFERÊNCIAS BIBLIOGRÁFICAS}

Albuquerque UP, Ramos MA, Ferreira Júnior WS, Medeiros, PM. 2017. Ethnobotany for Beginners. Gewerbestrasse: Springer International Publishing, 71p.

Albuquerque UP, Ramos MA, Lucena RFP, Alencar NL. 2014. Methods and Techniques Used to Collect Ethnobiological Data. In: Albuquerque UP, Cunha LVFC, Lucena RFP, Alves RRN. Methods and Techniques in Ethnobiology and Ethnoecology. Humana Press, New York, p. 15-37.

Amaral CN. 2008. Recursos vegetais dos tradicionais quintais de Rosário Oeste - Mato Grosso. Dissertação (mestrado) Cuiabá, UFMT.

Amaral CN, Guarim-Neto G. 2008. Os quintais como espaços de conservações e cultivo de alimentos: um estudo na cidade de Rosário Oeste (Mato Grosso, Brasil). Bol. Mus. Para. Emílio Goeldi. Ciências humanas, 3(3):329-341.

Amaral CN, Guarim VLMS. 2007. Estudo Etnobotânico da comunidade rural de Cascavel (Jangada, MT). Revista Brasileira de Biociências, 5(supl. I):894-896.

Amorozo MCM. Sistemas Agrícolas tradicionais ea conservação deagrobiodiversidade. Disponível em: < http: homologa.ambiente.Sp.gov.br /EA /adm/ admarqs / Maria A. pdf $>$. Acesso 11. fev. 2010.

Carneiro DB, Barboza MSL, Menezes MP. 2010. Plantas nativas úteis na vila dos pescadores da reserva extrativista marinha Caeté - Taperaçu, Para, Brasil. Acta bot. Bras. 24(4): 1027 - 1033.

Carniello MA, Cruz MAB, Silva RS. 2008. Composição florística e sua utilização em quintais urbanos de Mirassol D'Oeste. In: Guarim-Neto G, Carniello MA. Quintais 
Matogrossense: Espaços de conservação e reprodução de saberes. Cáceres-MT, Editora Unemat.

Carniello MA, Silva RS, Cruz MAB, Guarim Neto, G. 2010. Quintais urbanos de Mirassol D’Oeste-MT, Brasil: uma abordagem etnobotânica. Acta Amazônia, 40(3): 451-470.

Cunha HF, Vale MS, Silva Junior CA, Campos RF, Carlos LO. 2007. Conhecimento empírico dos moradores da comunidade do entorno do Parque Municipal da Cachoeirinha (Iporá-Goiás). Acta Sci. Biol. Sci. 29(2): 203-212.

Damasceno AA, Barbosa AAA. 2008. Levantamento etnobotânico de plantas do bioma Cerrado na comunidade de Martinésia, Uberlândia, MG. Horizonte Científico, 2(1): p.8.

Fonseca GP. 2008. Centro Histórico de Poconé - reflexos da cultura da cultura pantaneira. In: Higa TCCS (org.). Estudos regionais sul - americanos: sociocultural, economia e dinâmica territorial na área central do continente. Cuiabá: Ed. UFMT.

Fiuza, A. G. 2003. Águas do Pantanal mediatizando a educação ambiental. Dissertação (mestrado) UFMT, Cuiabá.

Governo do Estado de Mato Grosso, Mato Grosso e seus municípios. Disponívelem:<http://www.mteseusmunicipios.com.br/NG/conteudo. php?sid=199\&cid=937>Acesso em: 10 . fev.2010.

Guarim-Neto G. 2008. Arbustos de árvores dos quintais tradicionais de Rosário Oeste: Observando a representação popular. In: Guarim-Neto G, Carniello MA. Quintais Matogrossense: Espaços de conservação e reprodução de saberes. Cáceres-MT, Editora Unemat.

Guarim Neto G, Amaral CN. 2010. Aspectos Etnobotânicos de quintais tradicionais dos moradores de Rosário Oeste, Mato Grosso, Brasil, Polibotânica, 29: 191-212.

Hanazaki N. 2003. Comunidades, conservação e manejo: o papel do conhecimento ecológico local. Biotemas, Florianópolis, v. 16, n. 1, p. 23-47.

Lorenzi H, Sartori SF, Bacher LB, Lacerda MTC. 2006. Frutas Brasileiras e exóticas cultivadas (de consumo in-natura). São Paulo: Instituto Plantarum de Estudos de Flora.

Magalhães A. 2006. Perfil Etnobotânico e conservacionista das Comunidades do entorno da reserva natural Serra das Almas, Ceará - Piauí, Brasil. Dissertação 
(Mestrado) Programa regional de Pós - Graduação em Desenvolvimento e Meio Ambiente, UFC: Fortaleza.

Mauro R. 2002. Estudos Faunísticos na Embrapa Pantanal. Arch. Zootec. 51(193): 193194 .

Moraes AS, Sampaio Y, Seidl A. 2009. Quanto Vale o Pantanal? A Valoração Ambiental Aplicada ao Bioma Pantanal. Dados eletrônicos - Corumbá: Embrapa Pantanal. Disponível em: http://www.cpap.embrapa.br/publicacoes/online/DOC105.pdf> Acessado: 09/02/2011 às 10h17min.

Morais FF. 2008. Conhecimento Ecológico Tradicional de Plantas Pelos Pescadores da Comunidade de Estirão Cumprido, Barão de Melgaço Pantanal Mato-Grossense. Dissertação (Mestrado em Ecologia e Conservação da Biodiversidade). Instituto de Biociências, Universidade Federal de Mato Grosso, Cuiabá. 69 p.

Morais FF, Morais RF, Silva CJ. 2009. Conhecimento ecológico tradicional sobre plantas cultivadas pelos pescadores da comunidade Estirão Comprido, Pantanal matogrossense, Brasil. Bol. Mus. Para. Emílio Goeldi. Cienc. Hum. 4(2):277-294.

Morais RF. 2006. Conhecimento Ecológico tradicional da pesca pela comunidade de Cuiabá Mirim- Barão de Melgaço, Pantanal Mato-Grossense, Mato Grosso. Dissertação (Mestrado em Ecologia e Conservação da Biodiversidade) Instituto de Biociências, Universidade Federal de Mato Grosso, Cuiabá. 8of.

Moura, CL, Andrade, LHC. 2007. Etnobotânica em Quintais Urbanos Nordestinos: um Estudo no Bairro da Muribeca, Jaboatão dos Guararapes - PE. Revista Brasileira de Biociências, Porto Alegre, v. 5, supl. 1, p. 219-221.

Pasa MC. 2007. Um olhar etnobotânico sobre as Comunidades do Bambá, Cuiabá: Entrelinhas: Ed. UFMT.

Pasa MC. 2004. Etnobiologia de uma comunidade ribeirinha no Alto da Bacia Aricá Açu, Cuiabá, Mato Grosso, Brasil.. Tese (Doutorado em Ciências Área de Concentração em Ecologia e Recursos Naturais)-Centro de Ciências Biológicas e da Saúde, Universidade Federal de São Carlos, São Carlos, 174 p.

Paula Filho WL. 2010. Caracterização Socioambiental da Microbacia do Ribeirão Piancó no Município de Anápolis (GO): Subsídios para Políticas Públicas, Desenvolvimento Sustentável e Educação Ambiental. Dissertação (mestrado) - Programa de PósGraduação em Sociedade, Tecnologia e Meio Ambiente Anápolis: Centro Universitário de Anápolis - Uni Evangélica. 
Richardson RJ. 2008. Entrevista. In: Pesquisa Social: Métodos e Técnicas.3.ed.São Paulo:Atlas:207-218.

Ritter MR, Silva TC, Araújo EL, Albuquerque UP. 2015. Bibliometric analysis of ethnobotanical research in Brazil (1988-2013). Acta Botanica Brasilica, 29(1):113-119.

Panyadee P, Balslev H, Wangpakapattanawong P, Inta A. 2018. Karen Homegardens: Characteristics, Functions, and Species Diversity. Econ Bot 72(3): 285-302.

Pilla MAC, Amorozo MCM. 2009. O conhecimento sobre os recursos vegetais alimentares em bairros rurais do Vale do Paraíba, SP, Brasil. Acta bot. bras. 23(4) 1190 $-1201$.

Reis SLA, Guarim Neto G. 200o. Porto Brandão: uma comunidade inserida no Pantanal de Barão de Melgaço - sua história, seus valores e sua gente In: III simpósio sobre recursos naturais e sócio-econômicos do Pantanal o desafios do novo milênio, Corumbá-MS.

Rosa LS, Silveira EL, Santos MM, Modesto RS, Perote JRS, Vieira TA. 2007. Os quintais agroflorestais em áreas de agricultores familiares no município de Bragança-PA: composição florística, uso de espécies e divisão de trabalho familiar Rev. Bras. de Agroecologia. 2(2).

Rosa LS, Vieira TA, Pires HCG. 2009. Quintais agloflorestais em comunidade rurais de Bonito, Pará. Rev. Bras. de Agroecologia, 4(2).

Santos, S. 2004. Um estudo etnoecológico dos quintais da cidade de Alta FlorestaMT Dissertação (mestrado) - Universidade Federal de Mato Grosso, Instituto de Biociências, 166 p.

Santos S, Guarim Neto G. 2008. Etnoecologia de quintais: Estrutura e diversidade de usos de recursos vegetais em Alta Floresta. In: Guarim-Neto G, Carniello MA. Quintais Matogrossense: Espaços de conservação e reprodução de saberes. CáceresMT, Editora Unemat.

Santos TM, Carnielo MA. 2017. Quintais produtivos nas unidades familiares dos assentados In: Castrillon SI, Puhl JI, Morais FF, Lopes AAETM. Escassez Hídrica e Restauração Ecológica no Pantanal: Recuperação das nascentes e fragmentos de mata ciliar do córrego no Assentamento Laranjeira I e mobilização para conservação dos recursos hídricos no Pantanal mato-grossense, São Paulo: Carlini \& Caniato, p. 147152. 
Santos, E G, Santos, SS, Vandermond, NG, Bartolomeu, IS, e Lucena, RFP. 2019. Utilização de recursos vegetais em áreas de quintais em uma comunidade rural localizada no entorno do Parque Nacional de Sete Cidades, Piauí, Nordeste do Brasil. Revista Brasileira de Gestão Ambiental e Sustentabilidade: vol. 6, n. 13, p. 365-383.

Silva MP, Mauro R, Mourão G, Coutinho M. 200o. Distribuição e quantificação de classes de vegetação do Pantanal através de levantamento aéreo. Revta brasil. Bot., $23(2): 143-152$.

Silva JS. 2010. Análise sócio - espacial e conhecimento etnobotânico em uma comunidade quilombola no Sudoeste de Goiás. Dissertação (Mestrado), Universidade Federal de São Carlos, $169 f$.

Souza LF. 2007. Recursos vegetais usados na medicina tradicional do Cerrado (comunidade de Baús, Acorizal, MT, Brasil). Rev. Bras. Pl. Med., 9(4):44-54.

Souza CA, Lani JL, Sousa JB. Origem e evolução do Pantanal Mato- Grossense. In: Vl Simpósio nacional de geomorfologia/ Regional conference on geomorphology, Brasil, Goiânia-GO, 6 a 10 de setembro de 2006.

Spiller C, Coelho MFB, Gonçalves VD, Piton LP, Camili EC. 2016. Estudo etnobotânico em quintais agroflorestais em bairro na cidade de Cuiabá, Mato Grosso. Revista Verde de Agroecologia e Desenvolvimento Sustentável. 11(5):138-142.

Tuler AC. 2011. Levantamento Etnobotânico Na Comunidade Rural De São José Da Figueira, Durandé, Mg, Brasil. Porto Alegre, 2011. Apresentada a Universidade Federal do Espírito Santo.

Valadão LM, Amorozo MCM, Motta DG. 2006. Produção de alimentos na unidade domiciliar, dieta e estado nutricional: A contribuição dos quintais em um assentamento rural no Estado de São Paulo. In: Albuquerque UP, Almeida CFCBR. Tópicos em conservação e etnobotânica de plantas alimentícias. Recife: Nupeea, p. 93-118.

Whitney CW, Luedeling E, Hensel O, Tabuti JRS, Krawinkel M, Gebauer J, Kehlenbeck K. 2018. The Role of Homegardens for Food and Nutrition Security in Uganda. Hum Ecol. 46:497-514. 